\title{
Automutilação em adolescentes: revisão integrativa da literatura
}

\author{
Self-mutilation among adolescents: an integrative review of the \\ literature
}

Érika de Sene Moreira (https://orcid.org/0000-0001-7744-9293) ${ }^{1}$

Raquel Rosa Mendonça do Vale (https://orcid.org/0000-0003-2089-8842) ${ }^{2}$

Camila Cardoso Caixeta (https://orcid.org/0000-0003-2479-408X) ${ }^{3}$

Ricardo Antonio Gonçalves Teixeira (https://orcid.org/0000-0002-1603-2088) ${ }^{1}$

\footnotetext{
${ }^{1}$ Programa de Pós-

Graduação em Saúde

Coletiva, Universidade

Federal de Goiás (UFG). R.

$235 \mathrm{~s} / \mathrm{n}$, Setor Universitário.

74605-050 Goiânia GO

Brasil.

erikasenew@outlook.com

${ }^{2}$ Centro Universitário

Mineiros, Unifimes.

Trindade GO Brasil.

${ }^{3}$ Faculdade de Enfermagem,

UFG. Goiânia GO Brasil.
}

\begin{abstract}
This study involves an integrative review of the literature regarding the knowledge produced by the scientific community about self-mutilation among adolescents in the period between January 2012 and June 2017. A search was conducted in the SCIELO and CAPES Portal databases in Portuguese, English and Spanish with a final sample of 71 articles. This study revealed a large number of publications at international level, with an emphasis on epidemiological articles and deficiency in the publication of intervention studies that investigate the efficacy of therapies and prevention programs.

Key words Self-mutilation, Adolescents
\end{abstract}

Resumo Trata-se de uma revisão integrativa da literatura acerca dos conhecimentos produzidos pela comunidade científica sobre automutilação em adolescentes no período de janeiro de 2012 a junho de 2017. A busca foi realizada nas bases de dados SCIELO e Portal CAPES, em português, inglês e espanhol com amostra final de 71 artigos. Este estudo mostrou grande número de publicações a nivel internacional, com destaque para artigos epidemiológicos e fragilidade na publicação de estudos de intervenção que investiguem a eficácia de terapias e programas de prevenção.

Palavras-chave Automutilação, Adolescentes 


\section{Introdução}

A adolescência é um período do desenvolvimento humano contido entre a infância e a fase adulta, marcada por mudanças físicas, cognitivas, emocionais, sociais e comportamentais ${ }^{1}$. Passar por essas transformações com sucesso e suporte familiar dos seus respectivos pares resulta em uma identidade adulta saudável e autônoma ${ }^{2}$.

A automutilação é um fenômeno que se inicia na adolescência, sendo considerado um fator de dificuldade para o desenvolvimento positivo desta etapa da vida ${ }^{3}$. As investigações sobre automutilação descrevem um fenômeno complexo e com grande variação quanto a nomenclatura, conceito, prevalência, possível origem e determinantes ${ }^{4}$.

Um estudo, realizado em sete países da Europa, definiu automutilação como comportamentos não fatais em que o indivíduo intencionalmente causa lesões a si mesmo provocando cortes, arranhões ou queimaduras na própria pele, salta de locais relativamente altos, ingere fármacos em doses superiores as posologias terapêuticas, faz uso de drogas ilícitas ou substâncias psicoativas com propósito de autoagressão e ingestão de substâncias ou objetos não ingerivéis ${ }^{5}$.

Esta revisão tem como objetivo apresentar uma síntese das produções científicas a respeito da automutilação em adolescentes no período de janeiro de 2012 a junho de 2017.

\section{Métodos}

Trata-se de uma revisão integrativa da literatura acerca da automutilação em adolescentes. O método possibilita a análise e síntese de pesquisas científicas de forma rigorosa e ampla, o que facilita a divulgação da ciência e promove a constatação de lacunas do conhecimento que precisam ser preenchidas com novas pesquisas ${ }^{6}$.

A pesquisa utilizou o seguinte percurso metodológico: 1) identificação do tema e seleção da questão norteadora; 2) estabelecimento de critérios para inclusão e exclusão dos estudos; 3 ) definição das informações a serem extraídas dos estudos selecionados; 4) avaliação metodológica dos estudos incluídos; 5) 51 interpretação dos resultados; 6) apresentação da revisão e síntese do conhecimento ${ }^{6}$.

A pergunta que norteou todo o processo de pesquisa foi: Como a automutilação em adolescentes tem sido discutida pela comunidade científica?
A amostra foi selecionada a partir da composição de estratégias de buscas on-line, mais especificamente nas bases de dados do Scientific Electronic Library Online (SCIELO) e no Portal de Periódicos CAPES, no mês de junho de 2017. Foram empregados os descritores automutilação e adolescentes e os seus correspondentes em língua inglesa, self-mutilation e adolescents, sendo estes indexados no sistema de Descritores em Ciências da Saúde (DeCS). Os descritores foram combinados com a utilização do operador booleano AND.

Os critérios de inclusão estabelecidos foram: artigos publicados entre janeiro de 2012 e junho de 2017, em português, inglês e espanhol e revisados por pares, totalizando 992 publicações. Os critérios de exclusão foram constituídos por meio da eliminação dos artigos do tipo revisão e reflexão e dos artigos que utilizaram amostras mistas (adolescentes e adultos jovens). Foram considerados adolescentes, os indivíduos de 10 a 21 anos, segundo definição cronológica de Steinberg ${ }^{7}$. Após aplicação dos critérios de inclusão e exclusão, foram selecionados 93 artigos.

Os artigos selecionados foram organizados a partir dos seguintes dados: título do artigo, autores, país de origem do estudo, delineamento do estudo, objetivo, instrumentos utilizados, nomenclatura utilizada para automutilação, amostra e resultados alcançados.

A leitura e avaliação metodológica dos 93 artigos foi realizada de forma independente pelos autores deste estudo, sendo utilizado de forma adaptada o instrumento do Critical Appraisal Skills Programme (CASP) ${ }^{8}$. O CASP contempla os seguintes itens: 1) objetivo claro e justificado; 2) metodologia adequada; 3 ) apresentação e discussão dos procedimentos metodológicos; 4) seleção adequada da amostra; 5) coleta de dados detalhada; 6) aspectos éticos; 7) análise de dados rigorosa e fundamentada; 8) apresentação e discussão dos resultados; 9) contribuições e indicações de novas questões de pesquisa.

Nos casos de discordância em relação a elegibilidade do artigo, foi discutido e resolvido por consenso entre os pesquisadores. Após a avaliação, 22 artigos foram excluídos, por não definirem a idade dos adolescentes, não apresentarem parecer de comitê de ética e por divulgarem resultados de pesquisa ainda não concluída. A amostra final constituiu-se de 71 artigos. (Figura 1).

Foram incluídos estudos com todos os tipos de delineamento, uma vez que a questão de pesquisa foi relativa à abrangência do conhecimento produzido acerca do tema investigado e não 
Figura 1. Fluxograma de seleção dos artigos.

se relacionava à eficácia de uma intervenção ou técnica.

Após todo o processo de leitura e análise dos dados, os mesmos foram organizados e estruturados em categorias emergidas a posteriori. Os resultados do estudo serão apresentados e discutidos a partir de nove categorias, sendo elas: caracterização da amostra; prevalência da automutilação; métodos e localização da automutilação; frequência e evolução da automutilação ao longo da vida; funções da automutilação; automutilação como diagnóstico autônomo e comorbidades; fatores de risco para automutilação; neurobiologia da automutilação; instrumentos de avaliação para automutilação e cuidados em automutilação.

\section{Resultados e discussão}

\section{Caracterização da amostra}

Dos 71 artigos, foi observada a seguinte quantidade de publicações por ano: 2012 - doze $\operatorname{artigos}^{9-20}, 2013$ - treze artigos ${ }^{21-33}, 2014$ - quatorze $\operatorname{artigos}^{34-47}, 2015$ - dezesseis artigos ${ }^{48-63}, 2016$ nove artigos ${ }^{64-72}$ e 2017 até o mês de junho - sete artigos publicados ${ }^{73-79}$.

Foram encontradas publicações de 23 países distintos, destacando que a distribuição geográfica dos trabalhos mostra concentração de estudos no continente Europeu (43,6\%). Na Europa foram realizados 31 estudos ${ }^{10-12,17,18,21,23,25,27,30,32-34}$ $36,38,40,41,46,47,49,54,56,60-64,66,68,71,73$ sendo que destes, três envolveram mais de um país ${ }^{33,60,73}$. Foram localizados 12 estudos nos Estados Unidos $^{9,15,16,19,20,26,29,44,51,58,66,74}$ e onze na China $^{13,22,24,28,31,50,52,55,69,72,78}$. A Austrália ${ }^{14,39,43,53,59,76}$ e Suécia ${ }^{25,27,30,32,36,49}$ realizaram seis estudos cada. A Alemanha três estudos ${ }^{10,34,38}$ independentes e outros dois em conjunto com a Suíça ${ }^{33,73}$. O Canadá ${ }^{48,57,65,75}$ realizou quatro estudos, Cingapu$\mathrm{ra}^{37,77,79}$, Israel ${ }^{35,45,70}$, Itáliaa ${ }^{12,18,41}$ e Portugal ${ }^{17,63,71}$ foram representados com 3 estudos cada. A Bélgica realizou 2 estudos independentes ${ }^{40,56}$ e um com os Países Baixos ${ }^{60}$. A Croácia ${ }^{11,61}$ e a Turquia ${ }^{46},{ }^{68}$ realizaram 2 estudos cada. Eslovênia ${ }^{64}$, Espanha $^{62}$, Finlândia $^{21}$, Inglaterra ${ }^{23}$, Irlanda ${ }^{67}$, México $^{42}$, Noruega $^{54}$ e Polônia ${ }^{47}$ um estudo.

$\mathrm{O}$ número de artigos encontrados nesta revisão demonstra o interesse da comunidade científica e a relevância do tema, visto a quantidade de publicações e a diversidade de países envolvidos. A América Latina não apresentou produções científicas nos últimos cinco anos ou realizou publicações em revistas sem indexação nas grandes bases.

Quanto ao idioma, apenas um estudo foi publicado em espanhol ${ }^{17}$, os demais foram publicados em inglês ${ }^{9-16,18-79}$, evidenciando a predominância da língua inglesa como mecanismo de divulgação das pesquisas pela comunidade científica.

Encontramos estudos com amostras comunitárias e clínicas. Em 45 estudos os participantes foram recrutados em escolas de Ensino Médio $^{13-18,21-25,27,28,30-32,36,38,39,41-43,45-47,49,50,52-60,62,63}$, 67,69,71,72,73,76-78, seguidos por instituições psiquiátricas e unidades de psiquiatria que apareceram como locais de execução de 19 estu$\operatorname{dos}^{10-12,19,26,29,33,34,44,48,51,61,64,66,70,73,74,75,79}$. Outros sete trabalhos recrutaram as amostras em locais diversos $^{9,20,35,37,40,65,68}$. De um modo geral, a concentração de estudos em escolas era esperada, uma 
vez que na adolescência é comum que os indivíduos se encontrem inseridos neste ambiente.

Em relação ao sexo dos participantes, 62 estudos apresentaram amostras de ambos os se$\operatorname{xos}^{11-32,34,36-39,41-60,62,63,65-69,72,73,74-79}$, havendo oito que incluíram apenas adolescentes do sexo feminino $9,10,33,35,40,64,70,73$ e um estudo com adolescentes do sexo masculino ${ }^{61}$. É notável a escassez de estudos sobre automutilação em adolescentes do sexo masculino.

Quanto as nomenclaturas para designar a automutilação, o termo Non-suicidal self-injury (NSSI) ${ }^{10,12-16,18,19,22,24-34,36-45,48-50,52,53,55-64,66,69,70,73-77}$ apareceu em 53 estudos, Self Injurious Behavior ${ }^{17,35,68,72,78}$ foi encontrado em cinco estudos, Self-Harm ${ }^{23,54,65,67}$ em quatro estudos, Deliberate Self-Harm ${ }^{51,71} \mathrm{em}$ dois estudos, Self-Cutting ${ }^{21}$, Cutting ${ }^{20}$, Autodestructive Behavior ${ }^{11}$, Cutaneus Deliberate Self-harm ${ }^{47}$, NonSuicidal Self-harm Behavior $^{46}$, Self-Harm Behavior ${ }^{79}$ e Self Inflict Injury ${ }^{9}$ aparecem em um estudo cada. A falta de consenso na definição da nomenclatura é evidente, entretanto Non-suicidal self-injury (NSSI) se caracterizou por uma terminologia usual pela representatividade nos estudos.

Em relação ao delineamento metodológico, a maioria dos estudos apresentam métodos de natureza quantitativa $a^{9-19,21-36,38-57,59-75,77-79}$ (67 artigos), sendo apenas dois artigos caracterizados como qualitativos $^{20,58}$ e 2 apresentando métodos mistos

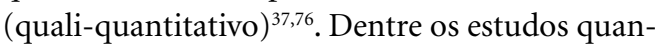
titativos, 56 apresentam delineamento transversal $^{9-12,14-19,21,22,24-26,29,32-36,38-42,44-49,51-54,56,59-66,68-75,77-79}$ e 11 apresentam delineamento longitudinal $^{13,23,27,28,30,31,43,50,55,57,67}$. A abordagem quantitativa traz a objetividade dos dados, taxas, indicadores, tendências e a abordagem qualitativa estuda a profundidade, subjetividade e complexidade do fenômeno, sendo ambas complementares ${ }^{80}$.

Entre as escalas e medidas padronizadas e validadas, a Avaliação Funcional da Automutilação (FASM) foi o instrumento adotado em 14 artigos $^{10,24,25,31-33,36-38,49,62,70,77,79}$ e o Inventário de Comportamentos de Auto Dano Deliberado (DSHI) em 6 estudos ${ }^{12,16,19,27,30,48}$.

\section{Prevalência da automutilação}

A prevalência de automutilação em adolescentes variou consideravelmente, indo de 10,1\% em um estudo realizado na Austrália ${ }^{39}$ a 75,9\%, taxa encontrada entre adolescentes internados em um hospital psiquiátrico de Cingapura ${ }^{79}$.

Entre os países que encontraram as menores taxas de prevalência estão Bélgica ${ }^{56}(13,4 \%)$,
Turquia $^{46}(14,4 \%)$, México ${ }^{42}(17,1 \%)$ e Polônia ${ }^{47}$ $(19,5 \%)$.

Além de Cingapura, as maiores prevalências foram encontradas entre os adolescentes espanhóis $^{62}(55,5 \%)$, seguidos de croatas ${ }^{61}(52,7 \%)$ em estudo realizado com adolescentes do sexo masculino, filhos de homens com diagnóstico de Transtorno de Estresse Pós-traumático.

Observa-se grande variação entre as taxas de prevalência, que podem estar relacionadas a falta de consenso na nomenclatura e conceito ${ }^{81}$, ao tipo de amostra (comunitária ou clínica) e aos aspectos geográficos e culturais ${ }^{4}$.

\section{Métodos e localização da automutilação}

A literatura aponta vários métodos e locais de automutilação sendo o corte citado como procedimento mais utilizado pelos adolescentes ${ }^{13,21}$.

Estudo realizado na Finlândia com 440 adolescentes que relatavam automutilação, indicou que a maioria $(67,2 \%)$ cortava apenas os braços, e que os adolescentes que cortam outras partes do corpo que não os braços apresentavam sintomas psicopatológicos mais sérios ${ }^{21}$. Da mesma forma, estudo realizado na China, evidenciou que o método utilizado para automutilação pode sinalizar diferentes psicopatologias associadas ${ }^{52}$.

O método de automutilação e o local apresentam diferenças relacionadas ao gênero ${ }^{15,67}$. Nos Estados Unidos, adolescentes do sexo feminino utilizavam os métodos de corte e arranhões e as lesões eram realizadas em braços e pernas. Já os adolescentes do sexo masculino usavam métodos mais agressivos como bater a cabeça e se queimar, provocar lesões nos peitos, no rosto e nos órgãos genitais ${ }^{15}$. A diferença evidente entre os métodos utilizados para automutilação por adolescentes do sexo feminino e masculino é a presença de sangue, as meninas tendem a usar métodos que envolvem sangramento enquanto os meninos não ${ }^{15}$.

Um estudo realizado na Irlanda relatou que a maioria das meninas que se automutilavam expressavam o desejo de "querer morrer", ao passo que entre os meninos o relato mais comum é que "queriam assustar alguém"67.

\section{Frequência e evolução da automutilação ao longo da vida}

A ocorrência da automutilação, em geral, segue padrões distintos nos adolescentes, podendo ser classificada de acordo com a frequência de episódios ao longo do tempo ${ }^{72}$. Estudo longitu- 
As funções da automutilação mais adotadas pelos adolescentes são as de Reforço Automático positivo (sentir alguma coisa, gerar sentimentos) e Reforço Automático Negativo (regular emoções negativas, como raiva, angústia, medo) $)^{37,77,25,31,45,20,79}$.

A análise de 100 narrativas sobre automutilação, postadas por adolescentes na Internet, sugere que por meio do pensamento mágico o adolescente processa e organiza emoções angustiantes utilizando o esquema corporal da automutilação ${ }^{20}$.

$\mathrm{Na}$ Suécia, estudo realizado com amostra comunitária de 816 adolescentes com idade entre 15 e 17 anos, indica que as experiências de abuso emocional e sintomas de depressão foram associadas à necessidade de se envolver na automutilação para regular emoções, autopunição ou gerar sentimentos. Já os sintomas de ansiedade previam funções sociais (comunicar dificuldades e facilitar agrupamento) $)^{36}$. Os adolescentes que sofreram abuso físico, tentaram suicídio e apresentavam sintomas de dissociação se envolveram em automutilação para atender tanto as funções automáticas como as funções sociais ${ }^{36}$.

Em Cingapura, um estudo realizado com adolescentes em hospital psiquiátrico indicou que a automutilação pode representar uma tentativa mal adaptada de gerenciar a desregulação emocional resultante da exposição a maus tra$\operatorname{tos}^{79}$.

Observamos que a maioria dos estudos caracteriza a automutilação predominantemente como função Automática Negativa, atribuindo a ela a regulação de emoções não desejadas e descaracterizando-a como um ato ou comportamento manipulador.

\section{Automutilação como diagnóstico autônomo e comorbidades}

Como sugerido pelo DSM-V, seis estudos indicam que a automutilação é um diagnóstico distinto ${ }^{33,22,26,34,66,73}$ caracterizado por alta comorbidade com depressão maior ${ }^{26,33}$, fobia social ${ }^{33}$, Transtorno de Estresse Pós-traumático ${ }^{33,58}$ Transtorno de Ansiedade ${ }^{26}$, comportamentos suici-

das $^{22,33}$, Transtornos Alimentares ${ }^{70}$ e Transtorno de Personalidade Boderline $e^{9,26,33,73}$.

A relação entre sintomas depressivos e automutilação aponta que, nos Estados Unidos, as adolescentes com automutilação apresentam maior desregulação emocional quando comparadas às adolescentes com Depressão ${ }^{9}$.

Sintomas depressivos são descritos por muitos adolescentes com automutilação ${ }^{12,28,57}$, entretanto, de acordo com estudo realizado na Bélgica e Países Baixos, as relações de automutilação e humor deprimido são menos pronunciadas em adolescentes que experimentam alto suporte parental ${ }^{60}$.

Três artigos associam automutilação e tentativa de suicídio ${ }^{29,32,59}$, evidenciando que os adolescentes que apresentavam automutilação associada a comportamentos de suicídio mostraram mais pontos de vista negativos sobre o eu, o mundo e o futuro, e têm percepção maior da falta de apoio parental ${ }^{29}$, relataram mais eventos adversos de vida e sintomas de traumas quando comparados com aqueles que apresentaram apenas automutilação ${ }^{32}$, além de apresentarem automutilação mais severa e altas taxas de vitimização $0^{59}$.

\section{Fatores de risco para automutilação}

Pertencer ao sexo feminino ${ }^{43}$, abuso físico ${ }^{18}$ e sexual ${ }^{18,35}$, Bullying ${ }^{60,75}$, consumo excessivo de álcool e drogas ${ }^{58}$, término de relacionamento ${ }^{58}$, baixa qualidade de relacionamento com a mãe ${ }^{18}$, falta de apoio familiar ${ }^{43,44,56}$, conhecer outra pessoa que se automutila ${ }^{28}$, sono pobre ${ }^{30}$, sintomas de impulsividade $e^{41,50}$, baixa autoestima ${ }^{43,44,53}$, baixo nível socioeconômico ${ }^{40}$, autocrítica ${ }^{50}$, dificuldade de resolução de problemas ${ }^{43}$, não possuir identificação religiosa ou espiritual ${ }^{43}$, baixa escolaridade $^{69}$, possuir identidade alternativa ${ }^{38}$, apresentar problemas com a lei ${ }^{68}$ e dificuldade de expressar emoções ${ }^{74}$ são considerados fatores de risco para o desenvolvimento da automutilação.

Um estudo realizado em Israel com 93 adolescentes do sexo feminino, de 12 a 18 anos, indicou que o abuso sexual na infância aumenta o risco de automutilação em três vezes ${ }^{35}$.

$\mathrm{Na}$ China, estudo realizado com 5787 adolescentes da comunidade examinou a socialização e a seleção de pares dos adolescentes automutiladores e identificou que o envolvimento dos melhores amigos com automutilação previu significativamente o comportamento de automutilação dos jovens. Além disso, os adolescentes com automutilação tendem a juntar-se a um grupo de pares também envolvidos com o comportamento ${ }^{28}$. 
Estudo realizado com adolescentes sob custódia, em uma prisão do Canadá, aponta que adolescentes do sexo feminino, com menor escolaridade e comportamentos diruptivos apresentam maior risco de desenvolver automutilação ${ }^{65}$.

Em Portugal, estudo realizado com 411 adolescentes de escolas do Ensino Médio mostrou que os adolescentes que se sentem desvalorizados e experimentam ameaças e submissão dentro da família tendem a relatar altos níveis de afeto negativo, o que, por sua vez, responde por maior envolvimento em automutilação ${ }^{71}$.

\section{Neurobiologia da automutilação}

Alguns estudos investigam a neurobiologia da automutilação. Um achado importante indica que o eixo hipotálamo-pituitária-adrenal dos adolescentes com automutilação é hiporresponsivo, portanto, a secreção de cortisol é reduzida e pode desempenhar fator de vulnerabilidade desses indivíduos no estresse agudo ${ }^{10}$.

Outro estudo sugere que as adolescentes com automutilação tomam decisões mais arriscadas e menos preocupadas com o resultado, se importando com ganhos imediatos, o que denota capacidade reduzida de aprender com decisões passadas. Este resultado indica que explorar os correlatos neurológicos da tomada de decisão pode melhorar a compreensão da automutilação $0^{64}$.

Por último temos um estudo sobre a capacidade de reconhecimento das emoções expressas pelo rosto, que é uma habilidade fundamental para interações sociais bem-sucedidas. Os déficits nesta capacidade podem fornecer informações sobre interações do comportamento cerebral. Este estudo identificou que os adolescentes com comportamento de automutilação cometiam mais erros de reconhecimento das emoções faciais ${ }^{66}$.

O estudo da neurobiologia da automutilação ainda é incipiente, porém acredita-se que o mesmo pode fornecer contribuições importantes na compreensão do mecanismo da automutilação.

\section{Instrumentos de avaliação para automutilação}

Os instrumentos para investigação da automutilação foram validados em cinco estudos, e outro estudo sugere o uso da avaliação breve das emoções negativas para identificar adolescentes com automutilação. Um destes foi realizado nos Estados Unidos e validou a Escala de Regulação de Dificuldades na Emoção (DERS). De acordo com os autores a DERS é uma ferramenta útil na detecção de automutilação em amostras clínicas de adolescentes ${ }^{19}$.

Outro estudo apontou a versão alemã da Entrevista de Pensamentos e Comportamentos Auto prejudiciais (SITBI-G) como instrumento apropriado para avaliar automutilação em adolescentes de acordo com os critérios sugeridos pelo DSM-V ${ }^{34}$.

O Inventário de Autolesão de Otawa (OSI) foi identificado como ferramenta válida e confiável para avaliação das funções da automutilação em adolescentes de amostras clínicas, com aplicação útil no planejamento de intervenções ${ }^{48}$.

O Questionário de Identidade, de Impulso, Auto ferimento e Suicídio para Adolescentes (ISSIQ-A) também foi validado como instrumento confiável para avaliar impulsos, automutilação e pensamentos suicidas na adolescência podendo ser usado na clínica e em pesquisa ${ }^{63}$.

Estudo realizado na Croácia examinou a relação entre automutilação e o modelo de cinco fatores da Personalidade (Traços de Personalidade Big Five). O estudo aponta que as dimensões da personalidade são importantes na avaliação do risco para automutilação em adolescentes e poderia ser utilizado para rastrear o ato em populações clínicas e não clínicas ${ }^{11}$.

Estudo na Inglaterra concluiu que a avaliação breve e direta das Emoções Negativas e da autoestima poderia ser usada para ajudar a identificar adolescentes em risco de automutilação em ambientes comunitários, uma vez que as ferramentas de rastreio questionam diretamente e muitos profissionais relutam em fazer perguntas diretas sobre $o$ assunto ${ }^{23}$.

\section{Cuidados em automutilação}

$\mathrm{Na}$ avaliação de terapias para automutilação, encontramos um estudo realizado nos Estados Unidos, que apresenta os resultados de um Programa de Terapia Comportamental Dialética, e considera esta abordagem eficaz para adolescentes com automutilação ${ }^{51}$.

Ao identificar os pontos de vista dos adolescentes sobre como pares e amigos on-line podem ajudar os jovens com automutilação, estudo realizado na Austrália, indicou como estratégias: falar sobre automutilação com colegas e amigos (mesmo em comunicação a distância), encaminhamento para adultos e profissionais de saúde, maior conscientização pública sobre automutilação e redução do estigma e bullying entre pares ${ }^{76}$.

Identifica-se carência de estudos que testem a eficácia de estratégias de prevenção e terapêuticas. 


\section{Considerações finais}

Este trabalho confirma a automutilação em adolescentes como um problema de saúde pública no mundo, uma vez que estudos de vários países trazem taxas altas e variadas de prevalência em amostras comunitárias e clínicas.

A complexidade do fenômeno é retratada pela diversidade de nomenclaturas e conceitos utilizados para descrevê-lo, o que se torna um fator de dificuldade para seu estudo.

O estudo demonstra evidências de que a automutilação é um diagnóstico autônomo que ocorre simultaneamente a outras comorbidades, sendo que, a literatura apresenta diversos fatores condicionantes e determinantes da automutilação em adolescentes, o que contribui para construção das hipóteses de tratamento e programas de prevenção. Entretanto estudos que testem a eficácia de abordagens de prevenção e terapêuticas são escassos.
Muitos são os estudos com finalidade de compreender as funções da automutilação e de validação de instrumentos que avaliem o comportamento, entretanto, percebe-se insuficiência de estudos que avaliem a trajetória da automutilação ao longo da vida.

Sugere-se o desenvolvimento de mais estudos: qualitativos e/ou de métodos mistos, longitudinais que avaliem a trajetória da automutilação e estudos que testem a eficácia de abordagens de prevenção e terapêuticas.

Não foram encontrados artigos de países da América Latina, como o Brasil, o que pode ser limitação da pesquisa que utilizou para busca bases de dados indexadas, bem como a falta de consenso da nomenclatura para automutilação. Esta restrição de dados nacionais aponta a necessidade de pesquisas com esta temática para conhecer a realidade local. Além disso, estudos epidemiológicos no Brasil são importantes para apresentar aos governos evidências que justifiquem políticas públicas e aloquem verbas para saúde mental infantojuvenil.

\section{Colaboradores}

ÉS Moreira: realizou mestrado sendo este artigo um dos produtos; participou da concepção e delineamento do estudo, coleta de dados, análise, interpretação de dados, redação do manuscrito e aprovação da versão final. RRM Vale: análise e interpretação de dados; redação do manuscrito; aprovação da versão final. CC Caixeta: oreintadora do estudo, concepção e delineamento do estudo; revisão crítica e aprovação da versão final. RG Teixeira: concepção e delineamento do estudo; revisão crítica e aprovação da versão final. 


\section{Referências}

1. American Psychological Association (APA). Developing Adolescents: A Reference for Professionals. Washington: APA; 2002.

2. Sampaio D. O Conceito de adolescência. In: Sampaio D, editor. Lavrar o Mar: um novo olhar sobre o relacionamento entre pais e filhos. Lisboa: Editorial Caminho; 2006. p. 17-25.

3. Cruz D. Viver com a vida, "morrer" com a vida: proteção e risco em trajectórias auto-destrutivas na adolescência. Lisboa: Universidade de Lisboa; 2013.

4. Hawton K, Saunders KE, O'Connor RC. Self-harm and suicide in adolescents. Lancet 2012; 379(9834):23732382.

5. Madge N, Hewitt A, Hawton K, Wilde EJD, Corcoran P, Fekete S, Heeringen KV, De Leo D, Ystgaard M. Deliberate self-harm within an international community sample of young people: comparative findings from the Child \& Adolescent Self-harm in Europe (CASE) Study. J Child Psychol Psychiatry 2008; 49(6):667-677.

6. Mendes KDS, Silveira RCCP, Galvão CM. Revisão integrativa: método de pesquisa para a incorporação de evidências na saúde e na enfermagem. Texto Contexto Enferm 2008; 17(4):758-764.

7. Steinberg L. Adolescence. New York: MacGraw-Hill; 1993.

8. Critical Appraisal Skills Programme (CASP) [Internet]. [acessado 2017 Set 01]. Disponível em: www. casp-uk.net/casp-tools-checklists

9. Crowell SE, Beauchaine TP, Hsiao RC, Vasilev CA, Yaptangco M, Linehan MM, McCauley E. Differentiating adolescent self-injury from adolescent depression: possible implications for borderline personality development. J Abnormal Child Psychol 2012; 40(1):45-57.

10. Kaess M, Hille M, Parzer P, Maser-Gluth C, Resch F, Brunner R. Alterations in the neuroendocrinological stress response to acute psychosocial stress in adolescents engaging in nonsuicidal self-injury. Psychoneuroendocrinology 2012; 37(1):157-161.

11. Kotrla Topić M, Perković Kovačević M, Mlačić B. Relations of the Big-Five personality dimensions to autodestructive behavior in clinical and non-clinical adolescent populations. Croatian Medical J 2012; 53(5):450-460.

12. Ferrara M, Terrinoni A, Williams R. Non-suicidal self-injury (Nssi) in adolescent inpatients: assessing personality features and attitude toward death. Child Adolescent Psychiatry Mental Health 2012; 6(1):12.

13. You J, Leung F, Fu K. Exploring the reciprocal relations between nonsuicidal self-injury, negative emotions and relationship problems in Chinese adolescents: A longitudinal cross-lag study. J Abnormal Child Psychol 2012; 40(5):829-836.

14. Andrews T, Martin G, Hasking P. (). Differential and common correlates of non-suicidal self-injury and alcohol use among community-based adolescents. Advances Mental Health 2012; 11(1):55-66.

15. Sornberger MJ, Heath NL, Toste JR, McLouth R. Nonsuicidal self-injury and gender: Patterns of prevalence, methods, and locations among adolescents. Suicide Life-Threatening Behav 2012; 42(3):266-278.
16. Howe-Martin LS, Murrell AR, Guarnaccia CA. Repetitive nonsuicidal self-injury as experiential avoidance among a community sample of adolescents. J Clinical Psychol 2012; 68(7):809-829.

17. Gonçalves SF, Martins C, Rosendo AP, Machado BC, Silva E. Self-injurious behavior in Portuguese adolescents. Psicothema 2012; 24(4):536-541.

18. Di Pierro R, Sarno I, Perego S, Gallucci M, Madeddu F. Adolescent nonsuicidal self-injury: the effects of personality traits, family relationships and maltreatment on the presence and severity of behaviours. Eur Child Adolesc Psychiatry 2012; 21(9):511-520.

19. Perez J, Venta A, Garnaat S, Sharp C. The difficulties in emotion regulation scale: factor structure and association with nonsuicidal self-injury in adolescent inpatients. J Psychopathol Behav Assessment 2012; 34(3):393-404.

20. Gregory RJ, Mustata GT. Magical thinking in narratives of adolescent cutters. J Adolesc 2012; 35(4):10451051.

21. Laukkanen E, Rissanen ML, Tolmunen T, Kylmä J, Hintikka J. Adolescent self-cutting elsewhere than on the arms reveals more serious psychiatric symptoms. Eur Child Adolesc Psychiatry 2013; 22(8):501-510.

22. Cheung YTD, Wong PWC, Lee AM, Lam TH, Fan YSS, Yip PSF. Non-suicidal self-injury and suicidal behavior: prevalence, co-occurrence, and correlates of suicide among adolescents in Hong Kong. Soc Psychiatry Psychiatr Epidemiol 2013; 48(7):1133-1144.

23. Phillips R, Spears MR, Montgomery AA, Millings A, Sayal K, Stallard P. Could a brief assessment of negative emotions and self-esteem identify adolescents at current and future risk of self-harm in the community? A prospective cohort analysis. BMC Public Health 2013; 13(1):604.

24. Tang J, Ma Y, Guo Y, Ahmed NI, Yu Y, Wang J. Association of aggression and non-suicidal self-injury: a school-based sample of adolescents. PloS One 2013; 8(10):e78149.

25. Zetterqvist M, Lundh LG, Dahlström Ö, Svedin CG. Prevalence and function of non-suicidal self-injury (NSSI) in a community sample of adolescents, using suggested DSM- 5 criteria for a potential NSSI disorder. J Abnorm Child Psychol 2013; 41(5):759-773.

26. Glenn CR, Klonsky ED. Nonsuicidal self-injury disorder: an empirical investigation in adolescent psychiatric patients. J Clin Child Adolesc Psychol 2013; 42(4):496-507.

27. Marshall SK, Tilton-Weaver LC, Stattin H. Non-suicidal self-injury and depressive symptoms during middle adolescence: a longitudinal analysis. $J$ Youth Adolesc 2013; 42(8):1234-1242.

28. You J, Lin MP, Fu K, Leung F. The best friend and friendship group influence on adolescent nonsuicidal self-injury. J Abnorm Child Psychol 2013; 41(6):9931004.

29. Wolff J, Frazier EA, Esposito-Smythers C, Burke T, Sloan E, Spirito A. Cognitive and social factors associated with NSSI and suicide attempts in psychiatrically hospitalized adolescents. J Abnorm Child Psychol 2013; 41(6):1005-1013. 
30. Lundh LG, Bjärehed J, Wångby-Lundh M. Poor sleep as a risk factor for nonsuicidal self-injury in adolescent girls. J Psychopathol Behavl Assessment 2013; 35(1): 85-92.

31. You J, Lin MP, Leung F. Functions of nonsuicidal self -injury among Chinese community adolescents. Journal Adolesc 2013; 36(4):737-745.

32. Zetterqvist M, Lundh LG, Svedin CG. A comparison of adolescents engaging in self-injurious behaviors with and without suicidal intent: self-reported experiences of adverse life events and trauma symptoms. Journal Youth Adolesc 2013; 42(8):1257-1272.

33. In-Albon T, Ruf C, Schmid M. Proposed diagnostic criteria for the DSM-5 of nonsuicidal self-injury in female adolescents: diagnostic and clinical correlates. Psychiatry J 2013; 2013:159208.

34. Fischer G, Ameis N, Parzer P, Plener PL, Groschwitz $\mathrm{R}$, Vonderlin E, Kaess M. The German version of the self-injurious thoughts and behaviors interview (SITBI-G): a tool to assess non-suicidal self-injury and suicidal behavior disorder. BMC Psychiatry 2014; 14(1):265.

35. Lev-Wiesel R, Zohar G. The role of dissociation in self-injurious behavior among female adolescents who were sexually abused. J Child Sexual Abuse 2014; 23(7):824-839.

36. Zetterqvist M, Lundh LG, Svedin CG. A cross-sectional study of adolescent non-suicidal self-injury: support for a specific distress-function relationship. Child Adolesc Psychiatry Mental Health 2014; 8(1):23.

37. Tan AC, Rehfuss MC, Suarez EC, Parks-Savage A. Nonsuicidal self-injury in an adolescent population in Singapore. Clinical Child Psychol Psychiatry 2014; 19(1):58-76.

38. Young R, Sproeber N, Groschwitz RC, Preiss M, Plener PL. Why alternative teenagers self-harm: exploring the link between non-suicidal self-injury, attempted suicide and adolescent identity. BMC Psychiatry 2014; 14(1):137.

39. Voon D, Hasking P, Martin G. The roles of emotion regulation and ruminative thoughts in non-suicidal self-injury. Br J Clinical Psychol 2014; 53(1):95-113.

40. Baetens I, Claes L, Martin G, Onghena P, Grietens H, Van Leeuwen K, Griffith JW. Is nonsuicidal self-injury associated with parenting and family factors? J Early Adolesc 2014; 34(3):387-405.

41. Di Pierro R, Sarno I, Gallucci M, Madeddu F. Nonsuicidal self-injury as an affect-regulation strategy and the moderating role of impulsivity. Child Adolesc Mental Health 2014; 19(4):259-264.

42. Delgadillo-González Y, Chávez-Flores CI, Martínez OL. Nonsuicidal self-injury in a community sample of older children and adolescents of Mexico City. Actas Esp Psiquiatr 2014; 42(4):159-168.

43. Andrews T, Martin G, Hasking P, Page A. Predictors of onset for non-suicidal self-injury within a school-based sample of adolescents. Prevention Sci 2014; 15(6):850-859.

44. Wolff JC, Frazier EA, Esposito-Smythers C, Becker SJ, Burke TA, Cataldo A, Spirito A. Negative cognitive style and perceived social support mediate the relationship between aggression and NSSI in hospitalized adolescents. J Adolesc 2014; 37(4):483-491.
45. Rodav O, Levy S, Hamdan S. Clinical characteristics and functions of non-suicide self-injury in youth. Eur Psychiatry 2014; 29(8):503-508.

46. Evren C, Evren B, Bozkurt M, Can Y. (). Non-suicidal self-harm behavior within the previous year among 10th-grade adolescents in Istanbul and related variables. Nordic J Psychiatry 2014; 68(7):481-487.

47. Gmitrowicz A, Kostulski A, Kropiwnicki P, ZalewskaJanowska A. Cutaneous Deliberate Self-harm in Polish School Teenagers-An Inter disciplinary Challenge. Acta Dermato-Venereologica 2014; 94(4):448-453.

48. Nixon MK, Levesque C, Preyde M, Vanderkooy J, Cloutier PF. The Ottawa Self-Injury Inventory: Evaluation of an assessment measure of nonsuicidal self-injury in an inpatient sample of adolescents. Child Adolesc Psychiatry Mental Health 2015; 9(1):26.

49. Dahlström Ö, Zetterqvist M, Lundh LG, Svedin CG. Functions of nonsuicidal self-injury: Exploratory and confirmatory factor analyses in a large community sample of adolescents. Psycholl Assessment 2015; 27(1):302.

50. You J, Lin MP, Leung F. A longitudinal moderated mediation model of nonsuicidal self-injury among adolescents. J Abnorml Child Psychol 2015; 43(2):381-390.

51. James S, Freeman KR, Mayo D, Riggs ML, Morgan JP, Schaepper MA, Montgomery SB. Does insurance matter? Implementing dialectical behavior therapy with two groups of youth engaged in deliberate self-harm. Adm Policy Ment Health 2015; 42(4):449-461.

52. You J, Ma C, Lin MP, Leung F. Comparing among the experiences of self-cutting, hitting, and scratching in Chinese adolescents attending secondary schools: An interview study. Behav Dis 2015; 40(2):122-137.

53. Tanner AK, Hasking P, Martin G. Non-suicidal self-injury and firesetting: shared and unique correlates among school-based adolescents. J Youth Adolesc 2015; 44(4):964-978.

54. Huang L, Mossige S. Resilience in young people living with violence and self-harm: evidence from a Norwegian national youth survey. Psychol Res Behav Manag 2015; 8:231.

55. Barrocas AL, Giletta M, Hankin BL, Prinstein MJ, Abela JR. Nonsuicidal self-injury in adolescence: Longitudinal course, trajectories, and intrapersonal predictors. J Abnorm Child Psychol 2015; 43(2):369-380.

56. Baetens I, Claes L, Hasking P, Smits D, Grietens H, Onghena P, Martin G. The relationship between parental expressed emotions and non-suicidal self-injury: The mediating roles of self-criticism and depression. J Child Family Studies 2015; 24(2):491-498.

57. Duggan J, Heath N, Hu T. Non-suicidal self-injury maintenance and cessation among adolescents: a one-year longitudinal investigation of the role of objectified body consciousness, depression and emotion dysregulation. Child Adolesc Psychiatry Mental Health 2015; 9(1):21.

58. Baker CK, Helm S, Bifulco K, Chung-Do J. The relationship between self-harm and teen dating violence among youth in Hawaii. Qualitative Health Res 2015; 25(5):652-667. 
59. Tanner AK, Hasking P, Martin G. Suicidality among adolescents engaging in nonsuicidal self-injury (NSSI) and firesetting: the role of psychosocial characteristics and reasons for living. Child Adolesc Psychiatry Mental Health 2015; 9(1):33.

60. Claes L, Luyckx K, Baetens I, Van de Ven M, Witteman C. Bullying and victimization, depressive mood, and non-suicidal self-injury in adolescents: the moderating role of parental support. J Child Family Studies 2015; 24(11):3363-3371.

61. Maršanić VB, Margetić BA, Bulić SO, Đuretić I, Kniewald H, Jukić T, Paradžik L. Non-suicidal self-injury among psychiatric outpatient adolescent offspring of Croatian posttraumatic stress disorder male war veterans: Prevalence and psychosocial correlates. Int J Soc Psychiatry 2015; 61(3):265-274.

62. Calvete E, Orue I, Aizpuru L, Brotherton H. Prevalence and functions of non-suicidal self-injury in Spanish adolescents. Psicothema 2015; 27(3):223-228.

63. Carvalho CB, Nunes C, Castilho P, Motta C, Caldeira S, Pinto-Gouveia J. Mapping non-suicidal self-injury in adolescence: Development and confirmatory factor analysis of the Impulse, Self-harm and Suicide Ideation Questionnaire for Adolescents (ISSIQ-A). Psychiatry Res 2015; 227(2):238-245.

64. Rigler T, Gosar D, Modic D. Decision-making in adolescent females who deliberately self-harm. Psihologija 2016; 49(1):87-103.

65. Casiano H, Bolton SL, Hildahl K, Katz LY, Bolton J, Sareen J. A population-based study of the prevalence and correlates of self-harm in juvenile detention. PLoS One 2016; 11(1): 0146918.

66. Seymour KE, Jones RN, Cushman GK, Galvan T, Puzia ME, Kim KL, Dickstein DP. Emotional face recognition in adolescent suicide attempters and adolescents engaging in non-suicidal self-injury. Eur Child Adolesc Psychiatry 2016; 25(3):247-259.

67. Rasmussen S, Hawton K, Philpott-Morgan S, O'connor RC. Why do adolescents self-harm? Crisis 2016; 37(3):176-183.

68. Ozsoy S, Kara K, Teke HY, Turker T, Congologlu MA, Sezigen S, Javan GT. Relationship between Self-Injurious Behaviors and Levels of Aggression in Children and Adolescents Who Were Subject to Medicolegal Examination. J Forensic Sci 2016; 61(2):382-387.

69. Zhang SC, Tao FB, Wu XY, Tao SM, Fang J. Low health literacy and psychological symptoms potentially increase the risks of non-suicidal self-injury in Chinese middle school students. BMC Psychiatry 2016; 16(1):327.

70. Itzhaky L, Shahar G, Stein D, Fennig S. In Eating-Disordered Inpatient Adolescents, Self-Criticism Predicts Nonsuicidal Self-Injury. Suicide Life-Threatening Behav 2016; 46(4):385-397.

71. Xavier A, Cunha M, Pinto-Gouveia J. The indirect effect of early experiences on deliberate self-harm in adolescence: Mediation by negative emotional states and moderation by daily peer hassles. J Child Family Studies 2016; 25(5):1451-1460.
72. Xin X, Ming Q, Zhang J, Wang Y, Liu M, Yao S. Four distinct subgroups of self-injurious behavior among Chinese adolescents: Findings from a latent class analysis. PloS One 2016; 11(7):e0158609.

73. Tschan T, Peter-Ruf C, Schmid M, In-Albon T. Temperament and character traits in female adolescents with nonsuicidal self-injury disorder with and without comorbid borderline personality disorder. Child Adolesc Psychiatry Mental Health 2017; 11(1):4.

74. Thomassin K, Marion CG, Venasse M, Shaffer A. Specific coping strategies moderate the link between emotion expression deficits and nonsuicidal self-injury in an inpatient sample of adolescents. Child Adolesc Psychiatry Mental Health 2017; 11(1):21.

75. Baiden P, Stewart SL, Fallon B. The mediating effect of depressive symptoms on the relationship between bullying victimization and non-suicidal self-injury among adolescents: Findings from community and inpatient mental health settings in Ontario, Canada. Psychiatry Res 2017; 255:238-247.

76. Berger E, Hasking P, Martin G. Adolescents' perspectives of youth non-suicidal self-injury prevention. Youth Society 2017; 49(1):3-22.

77. Ong SH, Tan ACY, Liang WZ. Functions of nonsuicidal self-injury in Singapore adolescents: Implications for intervention. Asian J Psychiatry 2017; 28:47-50.

78. Xin X, Wang Y, Fang J, Ming Q, Yao S. Prevalence and correlates of direct self-injurious behavior among Chinese adolescents: findings from a multicenter and multistage survey. J Abnorm Child Psychol 2017; 45(4):815-826.

79. Peh CX, Shahwan S, Fauziana R, Mahesh MV, Sambasivam R, Zhang Y, Subramaniam M. Emotion dysregulation as a mechanism linking child maltreatment exposure and self-harm behaviors in adolescents. Child Abuse Neglect 2017; 67:383-390.

80. Minayo MCS, Sanches O. Quantitativo-qualitativo: oposição ou complementaridade? Cad Saúde Pública 1993; 9(3):237-248.

81. Stanford S, Jones MP. How much detail needs to be elucidated in self-harm research? J Youth Adolesc 2010; 39(5):504-513.

Artigo apresentado em 16/07/2018

Aprovado em 22/01/2019

Versão final apresentada em 24/01/2019 